\title{
Interação Social e o Comportamento da Investidora Brasileira*
}

\author{
José Fajardo ${ }^{\dagger}$, Sandra Blanco ${ }^{\ddagger}$
}

Conteúdo: 1. Introdução; 2. Base de Dados; 3. Especificações; 4. Resultados; 5. Conclusões; A. Apêndice.

Palavras-chave: Ações, Mulheres, Investimentos, Comportamento, Risco.

Códigos JEL: G11, D03.

No presente artigo analisamos as características de mulheres que investem em ações no mercado brasileiro, seja diretamente, ou indiretamente, através de fundos de ações. Nosso objetivo é verificar se além dos fatores como idade, escolaridade, estado civil e patrimônio, há evidências de que a interação social aumenta a participação da mulher no mercado acionário. Através de um questionário enviado pela Internet para um grupo de investidoras, organizamos uma base de dados com variáveis determinantes na tomada de decisão de investimentos e analisamos as significâncias destas nas diversas combinações de regressões realizadas. Aplicamos o método Probit e calculamos os efeitos marginais sobre a variável dependente. Encontramos evidências que além do patrimônio financeiro, o nível de escolaridade o estado civil, sintomas de depressão e alguns indicadores de interação social são estatisticamente significantes quando a mulher escolhe seus investimentos. Também, como proposto por vários outros estudos, a propensão ao risco é determinante na escolha dos investimentos.

In the present paper we analyze the characteristics of women that invest in the Brazilian capital market, directly or indirectly, through stock funds. Our goal is to verify if, in addition to the usual factors like age, years of education, marital status and wealth, there is evidence that social interactions increase the participation of women in capital markets. Through a questionnaire sent by e-mail to a group of women investors, we organize a data base with variables that determines the investment decision and analyze their

\footnotetext{
*Agradecemos os comentários dos pareceristas e em especial os comentários do editor associado que ajudaram a melhorar o presente trabalho. J. Fajardo agradece também o apoio financeiro do CNPq do Brasil. Erros remanescentes são de responsabilidade exclusiva dos autores.

†Fundação Getulio Vargas, Escola Brasileira de Administração Pública e de Empresas (FGV/EBAPE). Praia de Botafogo 190, sala 534, CEP 22253-900. Rio de Janeiro, RJ. E-mail: pepefb@gmail . com

$\ddagger$ Ibmec Business School - RJ. E-mail: sandra.blanco@terra.com.br
} 
statistical significance, we use a Probity model and compute the marginal effects. We find evidence that additionally to wealth, years of education and marital status, depression and other indicators of social interaction are statistically significant when woman decides their investments. Also, as suggested by several works, risk propensity influences the investment decision.

\section{INTRODUÇÃO}

As características do gênero dos agentes nas tomadas de decisão tem sido analisadas por vários autores. Croson e Gneezy (2004) sugerem que a aversão ao risco pode levar a mulher a escolher profissões menos competitivas. Bajtelsmit e VanDerhei (1996), Hinz et alii (1996) encontraram que as mulheres investem seus planos de aposentadoria mais conservadoramente do que os homens. Das respostas de uma pesquisa com 5.200 homens e 6.400 mulheres, Barsky et alii (1995) concluem que as mulheres tem mais aversão ao risco. Jianakoplos e Bernasek (1998) argumentam que a aversão ao risco pode explicar porque as mulheres possuem níveis inferiores de riqueza quando comparados aos homens. Relataram que $60 \%$ das mulheres, contra somente $40 \%$ dos homens que participaram do Survey of Consumer Finances 1989, disseram não estar dispostas a enfrentar qualquer risco financeiro. As mulheres se dizem mais cautelosas ao investir seus recursos. Elas ponderam não ser fácil repor os recursos em caso de perdas e temem acabar a vida sem recursos, a chamada síndrome da Bag Lady. ${ }^{1}$

A participação da mulher no mercado de ações brasileiro parece suportar o estereótipo de que elas são mais avessas ao risco do que os homens, conforme dados da Tabela 1. Embora o aumento da participação das mulheres tenha sido mais acelerado em comparação aos dos homens nos últimos sete anos, passando de $17,63 \%$ em 2002, para $22,45 \%$ em 2008, esse percentual ainda é muito pequeno.

Tabela 1: Participação de investidores pessoa física no mercado de ações

\begin{tabular}{lccccc}
\hline & \multicolumn{2}{c}{ Homens } & \multicolumn{2}{c}{ Mulheres } & Total \\
\cline { 2 - 6 } & Qtd & $\%$ & Qtd & $\%$ & Qtd \\
\hline 2002 & 70.219 & 82,37 & 15.030 & 17,63 & 85.249 \\
2003 & 69.753 & 81,60 & 15.725 & 18,40 & 85.478 \\
2004 & 94.434 & 80,77 & 22.480 & 19,23 & 116.914 \\
2005 & 122.220 & 78,76 & 32.963 & 21,24 & 155,183 \\
2006 & 171.717 & 78,18 & 47.917 & 21,82 & 219.634 \\
2007 & 344.171 & 75,38 & 112.386 & 24,62 & 456.557 \\
2008 & 411,098 & 73,60 & 125.385 & 22,45 & 536.483 \\
\hline Fonte: Bovespa. & & & &
\end{tabular}

Do total de participantes pessoas físicas no mercado de ações no final de 2008 apenas $22,45 \%$ eram mulheres, mas observa-se um crescimento muito significativo nos últimos anos. Enquanto o aumento do total de participantes foi $529,31 \%$ de 2002 a 2008, o das mulheres foi $734,23 \%$. Embora crescente o número de investidores no Brasil é um número pequeno quando comparado ao mercado americano

${ }^{1}$ Pesquisa realizada pela companhia de seguro Allianz em agosto de 2006. 
Interação Social e o Comportamento da Investidora Brasileira

onde há 95 milhões de investidores pessoa física, sendo metade mulheres. De acordo com a pesquisa ${ }^{2}$ divulgada pela ION, ${ }^{3}$ de aproximadamente 1.000 mulheres que participaram, $85 \%$ são investidoras individuais de empresas de capital aberto e $84 \%$ investem através de fundo de ações.

Estudo 4 que analisou os dados do PNAD (Pesquisa Nacional por Amostra de Domicílios) entre os anos de 2004 e 2006, apontou que a participação da mulher cresceu em todos os segmentos. Enquanto a média do crescimento da população economicamente ativa (PEA) foi de $2,5 \%$ nesse período, o crescimento anual da $P E A^{5}$ feminina foi de $3,2 \%$ contra $1,9 \%$ da masculina.

Dados mais recente da Pesquisa Mensal de Emprego, em dezembro de 2008, havia 41,5 milhões de pessoas em idade ativa (com 10 anos ou mais de idade) no conjunto das seis regiões metropolitanas pesquisada e a PEA estimada em 23,7 milhões de pessoas. Na análise por sexo, as mulheres representavam a maioria da população em idade ativa $(53,4 \%)$, enquanto os homens $46,6 \%$, mas os homens continuavam a representar a maioria da população economicamente ativa $(54,2 \%)$.

A educação superior também tem sido um fator importante no progresso das mulheres. Elas representam $61 \%$ dos profissionais com nível superior, conforme o estudo citado e ainda segundo o Censo da Educação Superior de 2006 divulgado pelo Instituto Nacional de Estudos e Pesquisas Educacionais Anísio Teixeira (Inep), havia cerca de $12 \%$ mais mulheres que homens no ensino superior.

Até a tarefa de provedor da família não é mais exclusivamente masculina. A mulher assumiu a chefia da família, eram 18\% em 1991 e 33\% em 2007, segundo IBGE.

Então, se agora a mulher já tem educação superior, trabalha, tem uma renda e também é provedora, por que sua participação no mercado de ações ainda é tão pequena?

Recentemente, algumas explicações envolvendo o conceito de "interação social" tem sido sugeridas para explicar o comportamento dos investidores. Scheinkman (2005) define interação social como forma particular de externalidade na qual as ações de um grupo referencial afeta as preferências de um indivíduo.

No trabalho de Shiller (1995) há evidências de que pessoas que interagem umas com as outras regularmente tendem a pensar e a comportar-se de maneira semelhante. Especificamente para o mercado de ações, há pelo menos dois canais através dos quais a interação social pode influenciar a participação. O primeiro é o boca-a-boca ou aprendizado por observação ${ }^{6}$ (Banerjee, 1992, Bikhchandani et alii, 1992, Ellison e Fudenberg, 1995). Por exemplo, investidores potenciais podem aprender uns com os outros, tanto sobre os excepcionais retornos proporcionados historicamente pelo mercado, quanto sobre como executar operações. Depois, um investidor em ações pode sentir prazer em falar sobre os altos e baixos do mercado com os amigos, assim como sente prazer nas conversas sobre restaurantes, livros, cinema, esportes onde há interesses em comum, conforme observou Becker (1991). É preciso considerar também a cascata informacional, que ocorre quando um indivíduo toma decisão observando o comportamento dos indivíduos à sua frente sem levar em consideração sua própria informação. Bikhchandani et alii (1992) argumentam que o comportamento regional e a fragilidade dos comportamentos de massa podem ser explicadas por cascata informacional. Nas finanças uma possível aplicação é a decisão de investidores participarem de um IPO como fez Welch (1992) em seu estudo.

Mandrian e Shea (2001) exploraram uma variedade de esclarecimentos para os hábitos de poupança de empregados em grandes corporações americanas e, quase todos apontam para o fato de que mudanças economicamente significantes nos hábitos de poupar podem ser motivadas simplesmente pelo

\footnotetext{
2 “Women Investors Slow to Use Proxies to Change Board Composition”, Corporate Board Member Magazine On-Line. January 30, 2008.

3 InterOrganization Networking Advancing Women to the Boardroom - http://www. ionwomen. org/news.htm.

${ }^{4}$ Estudo realizado pelos professores Eugenia Troncoso Leone e Paulo Baltar, do IE (Instituto de Economia) da Unicamp http://www . bomdiariopreto. com.br/index. asp?jbd=1\&id=259\&mat=117899.

${ }^{5}$ Compreende as pessoas ocupadas mais as desocupadas.

${ }^{6}$ Hong et alii (2004).
} 
"poder da sugestão". Durlauf (2001) enfatiza como o contexto social e as interdependências sociais influenciam as maneiras como os indivíduos fazem escolhas.

Em trabalho recente, Hong et alii (2004) encontraram evidências de que a interação social aumenta a participação no mercado de ações, esse trabalho tem como objetivo principal testar empiricamente se a interação social é significante e avaliar os outros fatores determinantes exercendo influência sobre as escolhas de investimentos das mulheres.

Na nossa pesquisa seguiremos as noções de interação social usadas no trabalho de Hong et alii (2004) para verificar se alguma destas variáveis de interação social são determinantes na escolha da investidora Brasileira.

O trabalho foi estruturado da seguinte forma: na Seção 2 será apresentada a base de dados. Na Seção 3 serão apresentadas as especificações do trabalho, incluindo o case de dados e o modelo utilizado. Na Seção 4 serão apresentados os resultados e na última seção temos as Conclusões.

\section{BASE DE DADOS}

A base de dados do nosso estudo contém informações sobre mulheres com algum envolvimento no mercado acionário. Aproximadamente 1.000 mulheres foram convidadas a participar do estudo e receberam um questionário, descrito a seguir, por e-mail. Receberam e-mail as cadastradas do mailinglist do site Mulherinvest ${ }^{7}$ e/ou Programa Mulheres em Ação da Bovespa.

A base de dados para análise foi construída a partir de um questionário (em Apêndice). Esse questionário é uma versão simplificada do questionário utilizado no trabalho de Hong et alii (2004) da Health and Retirement Study (HRS) administrado pelo Institute for Social Research da University of Michigan. ${ }^{8}$

\subsection{Questionário}

O instrumento de coleta de dados utilizado neste estudo foi o questionário estruturado, composto por 25 (vinte e cinco) questões agrupadas nas seguintes classificações: dados pessoais, patrimônio, interação social, habilidades tecnológicas e outras características. Há tanto questões numéricas, quanto escalares, sim e não, múltipla escolha e de resposta breve.

As perguntas do questionário buscam extrair informações sobre educação, estado civil, renda, patrimônio, investimentos, aversão ao risco e também identificar o nível de sociabilidade e habilidades tecnológicas das participantes. A seguir apresentamos as principais características do perfil das participantes do estudo.

A idade média das participantes do estudo é de 42,1 anos. A maioria $(30,51 \%)$ se encontra na faixa entre 35 e 45 anos, seguida faixa de 45 a 55 anos $(29,89 \%)$.

O estado civil é uma variável importante na avaliação de perfil. A maioria das participantes é casada, $47,40 \%$, seguida pelas solteiras $27,92 \%$. O nível de escolaridade da participante também é uma variável importante na avaliação de perfil. Os níveis possíveis são: $1^{\circ}$ grau completo, $2^{\circ}$ grau completo, $3^{\circ}$ grau completo e pós-graduação. A maioria das participantes nesse estudo é pós-graduada, $56,49 \%$.

As participantes são na maioria profissionais, das mais variadas áreas do mercado de trabalho. São $94,80 \%$ as mulheres economicamente ativas. A profissão de cada uma foi identificada e a partir dessa informação criamos uma variável dummy para profissionais que de alguma forma mexem mais com dinheiro ou possuem alguma formação financeira como executivas, administradoras de empresa, economistas, comerciantes, que trabalham em vendas, bancárias, engenheiras e especialistas de marketing. São $32,46 \%$ as profissionais com essas qualificações. É intuitivo que essas profissionais se interessem mais pelo assunto do que as profissionais de áreas de humanas, médicas ou biológicas e artistas.

\footnotetext{
${ }^{7}$ http://www.bolsademulher.com.br/mulherinvest e Clube de Investimentos Mulherinvest.

${ }^{8}$ Disponiveis no site http://www. umich.edu/ ${ }^{\mathrm{hrswww} / .}$
} 
Interação Social e o Comportamento da Investidora Brasileira

Das participantes, apenas $38,96 \%$ trazem da infância lembranças de problemas financeiros. A ausência de problemas financeiros pode ser um indício de que a disciplina ou habito de investir tenha sido passado dos pais e/ou avós. Conforme proposto por Levy (2003) nós herdamos nossas preferências ou elas são determinadas randomicamente ou através de experiências? Mais fácil arriscar um dinheiro herdado do que de aquele que recebido pela renda de trabalho. Thaler (1999) denominou de Contabilidade Mental o processo sobre o qual as pessoas criam contas virtuais em suas mentes e atribuem gastos, poupança e riscos de forma diferente para cada uma dessas contas, muito embora os recursos sejam todos de uma mesma pessoa. Ainda Chiteji e Stafford (1999) documentaram que adultos são mais prováveis de participar do mercado de ações quando seus pais participaram.

É também importante identificar a localização da participante ou região onde mora. Essa questão é composta por duas perguntas de resposta breve: cidade e estado. Hong et alii (2004) concluíram que o impacto da sociabilidade é muito mais forte nos estados onde as taxas de participação no mercado de ações são mais elevadas. Diferentemente da base de dados americana, nesse estudo não vai ser possível explorar a variável localização. O mercado acionário brasileiro está fortemente concentrado nas grandes capitais do país. Dos investidores pessoas fisicas (homens e mulheres) que compram e vendem ações, a grande maioria com $74,14 \%{ }^{9}$ da participação, encontra-se no eixo São Paulo - Rio. Assim com a localização da maioria das participantes desse estudo, 83,11\%.

Também não vai ser possível explorar a variável raça das mulheres da amostra já que a maioria é predominantemente branca, 94,15\%. Hong et alii (2004) encontraram evidências que a participação dos brancos e não-hispânicos é maior do que a de outros grupos raciais.

Na segunda parte, as questões são referentes ao patrimônio das participantes. São cinco faixas de renda: abaixo de $R \$ 400$, de $R \$ 400$ a $R \$ 2.000$, de $R \$ 2.000$ a $R \$ 4.000$, de $R \$ 4.000$ a $R \$ 6.000$ e acima de $\mathrm{R} \$ 6.000$.

Também buscamos identificar o hábito de poupança da participante. Para os fins da análise o percentual da poupança mensal foi ignorado. Se há ou não o hábito de poupar mensalmente é o que interessa nesse estudo.

Pergunta relevante ao estudo é a identificação das alternativas de investimentos escolhidas pelas mulheres participantes. Cada alternativa é uma variável dummy e as possibilidades são: caderneta de poupança, $C D B$, fundo de renda fixa, ações - que incluem também fundo de ações ou clube de investimentos e outros investimentos. Se a mulher investe em ações diretamente ou indiretamente, via fundos, é indiferente. O que importa neste estudo também não é se a mulher está investindo mais ou menos em ações, apenas se investe, sim ou não e $67,80 \%$ da amostra são mulheres que investem em ações.

As outras formas de investimentos não incluídas nesse estudo foram identificadas com resposta breve. Dessas outras formas de investimentos as que mais apareceram foram: previdência privada, fundos multimercados, títulos de capitalização, Tesouro Direto, imóveis e dólares. No entanto, não foi possível inferir sobre as aplicações nessas outras formas de investimentos não abordadas pela pesquisa.

As faixas de patrimônio financeiro identificadas no estudo são: $\mathrm{R} \$ 5.000$, de $\mathrm{R} \$ 5.000$ a $\mathrm{R} \$ 25.000$, de $R \$ 25.000$ a $R \$ 50.000$, de $R \$ 50.000$ a $R \$ 75.000$ e acima de $R \$ 75.000$. A maioria das participantes possui um patrimônio financeiro acima de R\$ 75.000, são 57,79\%.

Pesquisas suportam a premissa que as variáveis descritas a seguir são boas medidas de interação informacional entre os indivíduos. No estudo de Granovetter (1983) é enfatizado "a força dos estranhos" que é a quantidade de informação que os indivíduos obtêm através de interação com vizinhos e conhecidos. Por exemplo, há fortes evidências para o efeito que os indivíduos desenvolvem mais novas habilidades através de conexões informais do que através de canais formais.

Pessoas que interagem por participarem de um grupo cuja religião seja o interesse em comum. Das participantes, $68,83 \%$ participam de algum grupo com essa característica.

\footnotetext{
${ }^{9}$ Fonte: Bovespa.
} 
Pessoas que praticam esportes podem interagir socialmente. Das participantes, 59,86\% praticam algum tipo de esporte. Participar de um clube ou academia também sugere uma forma de interação social. Das participantes, $62,83 \%$ frequentam clube ou academia e $64,42 \%$ participam de grupo ou associação.

Chegamos à quarta parte do questionário que é referente a outros traços de personalidade das participantes. Aqui se encontram questões elaboradas para criar proxies para esses traços de personalidade.

A primeira questão dessa parte quer identificar o perfil de risco da mulher. Também uma variável dummy e a resposta será 1 se a participante é propensa ao risco e 0 se for avessa ao risco. Essa questão foi elaborada por Barsky et alii (1995), no entanto, observou-se uma possível falha do questionário quanto à essa pergunta. Uma delas declarou que não entendeu o enunciado da questão, o que leva a concluir que muitas outras que também não responderam à questão, não o fizeram pelo mesmo motivo. Uma explicação para o problema dessa questão pode ser que as mulheres quando efetuam cálculos correm o risco de serem julgadas pelo estereótipo negativo de não terem habilidades matemáticas, Spencer et alii (1999).

Pode também ser por motivos de viés na avaliação de eventos conjuntivos e disjuntivos. As pessoas tendem a superestimar a probabilidade do evento conjuntivo e subestimar a probabilidade do evento disjuntivo. Os ganhos são menos valorizados do que as perdas, assim é fácil realizar trocas. Também por causa aversão ao risco no domínio dos ganhos (Barberis e Thaler, 2003).

Uma questão para identificar sintomas de depressão da participante, muito comum entre as mulheres. Variável dummy com resposta 0 se a participante nunca se sentiu deprimida ou 1 , se pelo menos de vez em quando apresentou algum sintoma de depressão. A premissa aqui é que há relação entre depressão e pessimismo, então as com sintomas de depressão não optariam por investimentos mais arriscados com as ações. A depressão também impacta o nível de interação social das participantes, já que é plausível que indivíduos mais deprimidos não interajam com outros. Das participantes, 55,19\% apresentam sintomas de depressão.

A última parte quer identificar as habilidades da participante com a tecnologia disponível. É importante identificar essas habilidades já que o avanço da tecnologia teve impacto nos hábitos de investimentos dos indivíduos. Choi et alii (2000) identificaram que o efeito Web é muito significativo, dobra a frequência das operações e o turnover da carteira cresceu mais de $50 \%$.

"A Internet também tem a capacidade de intensificar as relações sociais" segundo Amy Wells pesquisadora do Pew Internet \& American Life Project. ${ }^{10}$ Em seu estudo encontrou que $58 \%$ dos entrevistados têm na web fonte preferencial de busca de informação - seja ela para preenchimento do imposto de renda ou para um tratamento médico.

Considere as consequências das mudanças em tecnologia, por exemplo, o início das operações via homebroker, que permitiram os custos diretos da participação mais baixos para todos os investidores. Em muitos casos, esta mudança teve maior impacto na participação dos "sociais" do que na dos "nãosociais" por causa das externalidades positivas que os "sociais" conferem uns aos outros.

Com base nas respostas do questionário foi elaborada uma base de dados com as variáveis que se encontram no Quadro 1, as estatísticas destas variáveis são apresentadas na Tabela A-1 no Apêndice.

Um grande número de mulheres se recusou a participar do estudo, se negando a responder às questões mesmo que para fins acadêmicos. As pessoas não gostam de falar sobre sua situação financeira, principalmente por motivos de segurança. Dessa forma, os critérios de conveniência utilizados na amostra foram o interesse e a disponibilidade das mulheres em participar da pesquisa.

O procedimento ou fluxograma de coleta dos dados da pesquisa obedecem à seguinte sequência:

a) elaboração de uma carta de apresentação contendo os objetivos da pesquisa e as questões do estudo;

b) envio da carta de apresentação com o questionário; e

\footnotetext{
${ }^{10}$ Época Negócios - Edição 14 - Abril de 2008, 02/04/2008 - O mistério que ainda cerca a web 2.0 Por que as empresas ainda não estão tirando partido da internet como deveriam? Uma pesquisadora explica. Por Álvaro Oppermann.
} 
Quadro 1 - Resumo das variáveis utilizadas

\begin{tabular}{|c|c|c|}
\hline Variável & Nome & Possíveis conteúdos \\
\hline ACOES & Ações & 1 - investe em ações 0 - não investe em ações \\
\hline CPOUP & Caderneta de poupança & 1 - aplica em caderneta de poupança 0 - não aplica \\
\hline $\mathrm{CDB}$ & CDB ou Certificado de depósito bancário & 1 - compra de CDB 0 - não compra \\
\hline FRF & Fundos de Renda Fixa & 1 - aplica em fundos de renda fixa 0 - não aplica \\
\hline OUT & Outros & 1 - investe em outros produtos ou ativos 0 - não investe em outros \\
\hline IAB25 & Idade abaixo de 25 anos & 1 - faixa etária $<250$ - outra faixa \\
\hline I25A35 & Idade entre 25 a 35 anos & 1 - faixa etária $=[25,35] 0$ - outra faixa \\
\hline I35A45 & Idade entre 35 a 45 anos & 1 - faixa etária $=[23,45] 0$ - outra faixa \\
\hline I45A55 & Idade entre 45 a 55 anos & 1 - faixa etária $=[45,55] 0$ - outra faixa \\
\hline IAC55 & Idade acima de 55 anos & 1 - faixa etária $>=550$ - outra faixa \\
\hline SOL & Solteira & 1 - solteira 0 - não solteira \\
\hline CAS & Casada & 1 - casada 0 - não casada \\
\hline SEP & Separada & 1 - separada 0 - não separada \\
\hline VIU & Viúva & 1 - viúva 0 - não viúva \\
\hline FILHO & Filhos & 1 - se tem filhos 0 - se não tem filhos \\
\hline EDUC1 & Educação primária & 1 - nível de escolaridade primário 0 - tem outro nível de escolaridade \\
\hline EDUC2 & Educação secundária & 1 - nível de escolaridade secundário 0 - tem outro nível de escolaridade \\
\hline EDUC3 & Educação universitária & 1 - nível de escolaridade universitário 0 - tem outro nível de escolaridade \\
\hline EDUC4 & Pós-graduação & 1 - pós-graduação 0 - tem outro nível de escolaridade \\
\hline TRAB & Trabalho & 1 - trabalha 0 - não trabalha \\
\hline PROF & Profissão & 1 - trabalha 0 - não trabalha \\
\hline PROBFIN & Problemas financeiros na infância & $\begin{array}{l}1 \text { - enfrentou dificuldades financeiras na infância } 0 \text { - teve uma infância sem } \\
\text { problemas financeiros }\end{array}$ \\
\hline EST & Estado & 1 - está em São Paulo ou no Rio 0 - está em outro Estados \\
\hline COR & Raça & 1 - tem a cor branca 0 - tem outra cor \\
\hline R0 & Renda abaixo de $\mathrm{R} \$ 400$ & 1 - faixa de renda abaixo de $\mathrm{R} \$ 4000$ - outra faixa \\
\hline R0A2 & Renda entre $\mathrm{R} \$ 400$ e $\mathrm{R} \$ 2.000$ & 1 - faixa de renda entre $R \$ 400$ e $R \$ 2.0000$ - outra faixa \\
\hline $\mathrm{R} 2 \mathrm{~A} 4$ & Renda entre $\mathrm{R} \$ 2.000$ e R\$ 4.000 & 1 - faixa de renda entre $R \$ 2.000$ e $R \$ 4.0000$ - outra faixa \\
\hline R4A6 & Renda entre $R \$ 4.000$ e $R \$ 6.000$ & 1 - faixa de renda entre $R \$ 4.000$ e $R \$ 6.0000$ - outra faixa \\
\hline RAC6 & Renda acima de $\mathrm{R} \$ 6.000$ & 1 - faixa de renda acima de $R \$ 6.0000$ - outra faixa \\
\hline POUPO & Não tem hábito de poupança & 1 - não tem hábito de poupar mensalmente 0 - poupa mensalmente \\
\hline POUP1A5 & Poupa entre $1 \%$ e $5 \%$ da renda & 1-poupa entre $1 \%$ e $5 \% 0$ - poupa outra $\%$ \\
\hline POUP5A10 & Poupa entre $5 \%$ e $10 \%$ da renda & 1-poupa entre $5 \%$ e $10 \% 0$ - poupa outra $\%$ \\
\hline POUPAC10 & Poupa acima de $10 \%$ da renda & 1-poupa acima de $10 \% 0$ - poupa outra $\%$ \\
\hline PATRIF1 & Patrimônio financeiro até RS 5.000 & 1 - faixa de patrimônio financeiro abaixo de R\$ 5.0000 - outra faixa \\
\hline PATRIF2 & Patrimônio financeiro entre R\$ 5.000 e R\$ 25.000 & 1 - faixa de patrimônio financeiro entre $\mathrm{R} \$ 5.000$ e $\mathrm{R} \$ 25.0000$ - outra faixa \\
\hline PATRIF3 & Patrimônio financeiro entre $\mathrm{R} \$ 25.000$ e R\$ 50.000 & $\begin{array}{l}1 \text { - faixa de patrimônio financeiro entre } \mathrm{R} \$ 25.000 \text { e } \mathrm{R} \$ 50.0000 \text { - outra } \\
\text { faixa }\end{array}$ \\
\hline PATRIF4 & Patrimônio financeiro entre $\mathrm{R} \$ 50.000$ e R\$ 75.000 & $\begin{array}{l}1 \text { - faixa de patrimônio financeiro entre RS } 50.000 \text { e RS } 75.0000 \text { - outra } \\
\text { faixa }\end{array}$ \\
\hline PATRIF5 & Patrimônio financeiro acima RS 75.000 & 1 - faixa de patrimônio financeiro acima de R\$75.000 0 - outra faixa \\
\hline IMOV & Imóvel & 1 - possui imóvel 0 - não possui imóvel \\
\hline AUTO & Automóvel & 1 - possui automóvel 0 - não possui automóvel \\
\hline SOCIAL & Relacionamento com parentes e amigos & 1 - visita parentes ou amigos 0 - não visita \\
\hline VIZIN & Relacionamento com vizinhos & 1 - visita os vizinhos 0 - não visita \\
\hline RELIG & Religião & 1 - frequenta serviços religiosos 0 - não frequenta \\
\hline ESP & Esporte & 1 - pratica esportes 0 - não pratica \\
\hline CLUBE & Clube ou academia & 1 - frequenta clube ou academia 0 - não frequenta \\
\hline GRUPO & Grupo ou associação & 1 - participa de algum grupo ou associação 0 - não participa \\
\hline RISCO & Risco & 1 - corre riscos 0 - não corre riscos \\
\hline DEPRE & Sintomas de depressão & 1 - sentiu-se deprimida recentemente 0 - sentiu-se bem \\
\hline EMAIL & E-mail & 1 - tem conta de e-mail 0 - não tem conta de e-mail \\
\hline COMP & Computador & 1 - possui computador 0 - não possui computador \\
\hline
\end{tabular}


c) compilação das respostas recebidas.

Os e-mails com as perguntas foram enviados no fim de março de 2008 e recebemos cento e cinquenta e quatro respostas até o inicio do mês de maio.

\section{ESPECIFICAÇÕES}

A primeira parte do estudo visa contribuir para a resposta da seguinte questão: que fatores observáveis são significantes em mulheres investidoras em ações?

As variáveis idade, escolaridade, estado civil, e renda apontam para uma correlação com o risco dos investimentos. Os mais novos e solteiros arriscam mais. Aqueles com maior renda também são mais propensos a arriscar. Esses resultados estão fortemente de acordo com a noção do senso comum e com os resultados encontrados em Barber e Odean (2001).

Com base na literatura existente ${ }^{11}$ e no trabalho de Hong et alii (2004), os fatores determinantes na escolha dos investimentos são apresentados no Quadro 2.

Quadro 2 - Possíveis determinantes da escolha dos investimentos

\begin{tabular}{lc}
\hline Determinante & Código da variável \\
\hline Idade & IDADE \\
Estado Civil & ECIVIL \\
Renda & RENDA \\
Educação & EDUC \\
Risco & RISCO \\
Filhos & FILHO \\
Profissão & PROFIS \\
Região em que mora & EST \\
Raça ou Cor & COR \\
Patrimônio Financeiro & PATRIF \\
Percentual de Poupança & POUP \\
Propriedade de imóvel & IMOV \\
Propriedade de automóvel & AUTO \\
Social (Interação com parentes e/ou amigos) & SOCIAL \\
Interação com Vizinhos & VIZIN \\
Interação no Grupo Religioso & RELIG \\
Interação Clube ou Academia & CLUBE \\
Interação Grupo ou Associação & GRUPO \\
Sintomas de depressão & DEPRE \\
Tecnologia por e-mail & EMAIL \\
Tecnologia por computador & COMP \\
\hline
\end{tabular}

\footnotetext{
${ }^{11}$ Alguns fatos determinantes sobre a participação das famílias no mercado de ações são conhecidos e documentados por VissingJorgensen (2000) e Bertrand et alii (2000).
} 
Inicialmente, realizou-se uma série de regressões como forma de avaliar preliminarmente a relação entre as alternativas de investimentos e as variáveis utilizadas no estudo. Os coeficientes das equações finais utilizadas nesse estudo podem ser encontradas na Tabela 2.

Tabela 2: Possíveis determinantes dos investimentos

Coeficientes do Probit (Variável dependente $=1$, se a investidora escolhe "I", $e=0$ se ela escolhe diferente de " $I$ ". Onde $I$ será alternadamente Ações, Poupança e Outros Investimentos). No caso de $I=$ Ações foram usadas duas especificações adicionais que mostraram significância em algumas das variáveis de interação social. As colunas Média e s.d. são respectivamente, a média e o desvio padrão das variáveis amostrais

\begin{tabular}{|c|c|c|c|c|c|c|c|}
\hline & & Ações & & Poupança & Outros invest. & Média & s. d \\
\hline IDADE & 0.006840 & 0.008392 & 0.025447 & 0.016582 & -0.014560 & 42.10 & $(10,3000)$ \\
\hline RISCO & 0.249313 & 0.190638 & 0.223067 & $0.641839^{* *}$ & -0.160242 & 0.3591 & $(0,4814)$ \\
\hline CAS & -0.292359 & -0.338372 & -0.362242 & $-0.531965^{*}$ & 0.398962 & 0.4740 & $(0,5009)$ \\
\hline SEP & -0.444941 & -0.535621 & -0.579811 & -0.482021 & 0.013733 & 0.2077 & $(0,4070)$ \\
\hline VIU & -0.879258 & -1.114529 & -1.451743 & -1.021446 & 0.180115 & 0.0389 & $(0,1941)$ \\
\hline EDUC3 & $1.009464^{*}$ & $1.090624^{*}$ & 1.003559 & 0.419495 & 0.399289 & 0.3766 & $(0,4861)$ \\
\hline EDUC4 & $1.286734^{* *}$ & $1.381873^{* *}$ & $1.268289^{* *}$ & 0.066323 & 0.477602 & 0.5649 & $(0,4973)$ \\
\hline PATRIF2 & $0.867360^{*}$ & $1.009704^{* *}$ & $0.881540^{*}$ & 0.446878 & 0.282025 & 0.1168 & $(0,3223)$ \\
\hline PATRIF3 & $1.300415^{* *}$ & $1.376979^{* *}$ & $1.046573^{*}$ & 0.525045 & 0.003847 & 0.0844 & $(0,2789)$ \\
\hline PATRIF4 & $1.570154^{* * *}$ & $1.696179^{* * *}$ & $1.511271^{* * *}$ & -0.035110 & $1.318511^{* *}$ & 0.0909 & $(0,2884)$ \\
\hline PATRIF5 & $1.271390^{* * *}$ & $1.388311^{* * *}$ & $1.197566^{* *}$ & 0.285637 & 0.571340 & 0.5779 & $(0,4955)$ \\
\hline DEPRE & $-0.370146^{*}$ & $-0.376177^{*}$ & -0.218718 & 0.272758 & -0.004528 & 0.5519 & $(0,4989)$ \\
\hline RELIG & $0.590502^{* *}$ & $0.739840^{* *}$ & 0.366080 & $-0.578705^{* *}$ & 0.6883 & $(0,4646)$ & \\
\hline POPU1A5 & & & $0.888652^{* *}$ & 0.404669 & 0.785314 & 0.202703 & $(0.1866)$ \\
\hline POUP5A10 & & & $1.228188^{* * *}$ & 0.437372 & $0.849487^{*}$ & 0.263514 & $(0.1662)$ \\
\hline POUPAC10 & & & $1.699341^{* * *}$ & 0.439006 & $0.926594^{* *}$ & 0.385135 & $(0.1479)$ \\
\hline Intercepto & $-1.715618^{*}$ & $-2.064639^{* *}$ & $-3.767215^{* * *}$ & -1.820877 & $-1.706668^{*}$ & & \\
\hline $\begin{array}{l}\text { Amostra } \\
\text { ajustada }\end{array}$ & 153 & 153 & 153 & 153 & 153 & & \\
\hline $\begin{array}{l}\text { Observações } \\
\text { incluídas } \\
\text { depois dos } \\
\text { ajustes }\end{array}$ & 148 & 148 & 148 & 150 & 150 & & \\
\hline Convergência & 4 & 4 & 5 & 5 & 5 & & \\
\hline McFadden $\mathrm{R}^{2}$ & 0.149938 & 0.175900 & 0.272782 & 0.101656 & 0.143806 & & \\
\hline
\end{tabular}

A variável dependente é um indicador da alternativa de investimento, podendo ser ações, caderneta de poupança e outras alternativas. Números com * são significantes ao nível de $10 \%$, números com ${ }^{* *}$ são significantes ao nível de $5 \%$ e números com ${ }^{* * *}$ são significantes ao nível de $1 \%$. Os coeficientes foram estimados pelo método Probit. Convergência atingida após $4 / 5$ interações.

Porém, como já é sabido muitos dos problemas empíricos em finanças sofrem do problema de endogeneidade, no nosso caso o mais crítico foi o das variáveis patrimônio e renda, onde existe causalidade reversa. Nas outras variáveis este problema não foi significativo. Para evitar isto poderiam ser usadas variáveis instrumentais, porém preferimos omitir uma destas variáveis e deixar somente uma: a variável patrimônio. Pois, estamos mais interessados em ver a significância da variável interação social e não em exaurir todos os determinantes do perfil da investidora. Objetivo também de Hong et alii (2004), numa amostra maior sem diferença de gênero.

Em seguida, utilizando o modelo Probit, calculou-se o efeito marginal na média e o efeito marginal médio. Seguem abaixo as etapas realizadas para os cálculos dos efeitos marginais:

Sejam 


$$
\begin{aligned}
p & =F(Z) \\
f(Z) & =\frac{d p}{d Z}=\frac{1}{\sqrt{2 \pi}} e^{-\frac{1}{2} Z^{2}} \\
Z & =\beta_{1}+\beta_{2} X_{2}+\cdots \beta_{k} X_{k}
\end{aligned}
$$

$\log 0$

$$
\frac{\partial p}{\partial X_{i}}=\frac{d p}{d Z} \frac{\partial Z}{\partial X_{i}}=f(Z) \beta_{i}=\left(\frac{1}{\sqrt{2 \pi}} e^{-\frac{1}{2} Z^{2}}\right) \beta_{i}
$$

Por último, usando as médias amostrais dos $X_{i}$, calculamos o efeito marginal na média e usando o STATA obtivemos o efeito marginal médio. Ambos efeitos são reportados na Tabela 3.

\section{RESULTADOS}

A seguir apresentamos os resultados encontrados. Dividimos os resultados três partes, dependendo da variável dependente da regressão: ações, caderneta de poupança e outros investimentos. A variável dependente é uma variável dummy e que o método utilizado foi o Probit, conforme detalhado nas especificações.

O maior interesse no nosso estudo é analisar as características das mulheres que investem em ações, mas aproveitando as informações da base de dados foi possível analisar também o perfil das mulheres quando investem em Caderneta de Poupança ou outros investimentos. Nesse estudo, outros investimentos agrupam qualquer ativo escolhido pela investidora para gerar retornos financeiros como imóvel, dólar, previdência privada, etc.

\subsection{Ações}

Conforme os resultados da Tabela 2, a propensão ao risco (RISCO) não é um fator estatisticamente significante em nenhuma combinação de variáveis.

A IDADE também não é estatisticamente significante. Segundo Barber e Odean (2001) era esperado que as mais novas arriscassem mais.

Os coeficientes de ECIVIL não são estatisticamente significantes. Era esperado que as solteiras arriscassem mais, como as evidências no trabalho de Barber e Odean (2001).

Nas análises desse estudo o patrimônio financeiro (PATRIF) é estatisticamente significante e apresenta sinal positivo. Mulheres na faixa de R 5.000 a R 25.000 , apresentam $44,85 \%$, mais probabilidade de investir em ações. Na faixa de $\mathrm{R} \$ 25.000$ a $\mathrm{R} \$ 50.000$, em $53,25 \%$, de $\mathrm{R} \$ 50.000$ a $\mathrm{R} \$ 75.000$, em $76,89 \%$ e acima de $\mathrm{R} \$ 75.000$, em $60,93 \%$. Os efeitos marginais calculados para as mulheres que possuem investimentos em ações são encontrados na Tabela 3.

O fator educação (EDUC) também é estatisticamente significante. O sinal é positivo e quanto maior o nível de escolaridade, maior a probabilidade de investir em ações. Há $64,53 \%$ de probabilidade de mulheres com pós-graduação investirem em ações.

A variável RELIG é estatisticamente significante e com sinal positivo. Mulheres que interagem num grupo formado pela mesma religião apresentam 37.64\% maior probabilidade de investir em ações.

A interação com vizinhos, grupos e associações ou clubes e academias não são estatisticamente significantes, conforme resultados encontrados por Hong et alii (2004).

A variável DEPRE é estatisticamente significante e com sinal é negativo. Mulheres que responderam sentir algum sintoma de depressão diminuem a probabilidade de investir em ações em $11,13 \%$, como esperado, também de acordo com os resultados encontrados por Hong et alii (2004). Os demais fatores não foram estatisticamente significantes no nosso estudo. 
Tabela 3: Efeitos marginais

EMA e EMO são respectivamente, o Efeito Marginal na Média e Efeito Marginal Médio calculados a partir dos coeficientes Probit da Tabela 2. Em parênteses os erros padrões

\begin{tabular}{|c|c|c|c|c|c|c|}
\hline & \multicolumn{2}{|c|}{ Ações } & \multicolumn{2}{|c|}{ Poupança } & \multicolumn{2}{|c|}{ Outros invest. } \\
\hline & EMA & EMO & EMA & EMO & EMA & EMO \\
\hline IDADE & $\begin{array}{c}0.0129478 \\
(0.0079)\end{array}$ & $\begin{array}{c}0.0094727 \\
(0.0056)\end{array}$ & $\begin{array}{c}0.0075831 \\
(0.0067)\end{array}$ & $\begin{array}{c}0.0053312 \\
(0.0048)\end{array}$ & $\begin{array}{c}-0.0089987 \\
(0.0089)\end{array}$ & $\begin{array}{c}-0.0044531 \\
(0.0045)\end{array}$ \\
\hline RISCO & $\begin{array}{c}0.1134996 \\
(0.1443)\end{array}$ & $\begin{array}{c}0.0764324 \\
(0.0998)\end{array}$ & $\begin{array}{c}-0.2935164 \\
(0.1233)\end{array}$ & $\begin{array}{c}-0.1886558 \\
(0.0739)\end{array}$ & $\begin{array}{c}-0.0990367 \\
(0.1686)\end{array}$ & $\begin{array}{c}-0.047721 \\
(0.0799)\end{array}$ \\
\hline CAS & $\begin{array}{c}-0.184314 \\
(0.1732)\end{array}$ & $\begin{array}{c}-0.1322577 \\
(0.1240)\end{array}$ & $\begin{array}{c}-0.2432704 \\
(0.1434)\end{array}$ & $\begin{array}{c}-0.1686078 \\
(0.0988)\end{array}$ & $\begin{array}{c}0.2465765 \\
(0.1952)\end{array}$ & $\begin{array}{c}0.122245 \\
(0.0977)\end{array}$ \\
\hline SEP & $\begin{array}{c}-0.295016 \\
(0.2185)\end{array}$ & $\begin{array}{c}-0.2234188 \\
(0.1663)\end{array}$ & $\begin{array}{c}-0.2204307 \\
(0.1825)\end{array}$ & $\begin{array}{c}-0.1410469 \\
(0.1042)\end{array}$ & $\begin{array}{c}0.0084876 \\
(0.2545)\end{array}$ & $\begin{array}{c}0.0026982 \\
(0.1266)\end{array}$ \\
\hline VIU & $\begin{array}{c}-0.738667 \\
(0.4016)\end{array}$ & $\begin{array}{c}-0.5233427 \\
(0.1974)\end{array}$ & $\begin{array}{c}-0.4671127 \\
(0.3369)\end{array}$ & $\begin{array}{c}-0.2241308 \\
(0.0910)\end{array}$ & $\begin{array}{c}0.1113192 \\
(0.5015)\end{array}$ & $\begin{array}{c}0.0543396 \\
(0.2749)\end{array}$ \\
\hline EDUC3 & $\begin{array}{c}0.510625 \\
(0.3241)\end{array}$ & $\begin{array}{c}0.3474216 \\
(0.1889)\end{array}$ & $\begin{array}{c}0.1918373 \\
(0.2545)\end{array}$ & $\begin{array}{c}0.1339346 \\
(0.1905)\end{array}$ & $\begin{array}{c}0.2467786 \\
(0.4171)\end{array}$ & $\begin{array}{c}0.1325656 \\
(0.2182)\end{array}$ \\
\hline EDUC4 & $\begin{array}{c}0.6453234 \\
(0.3207)\end{array}$ & $\begin{array}{c}0.4581056 \\
(0.2035)\end{array}$ & $\begin{array}{c}0.0303298 \\
(0.2542)\end{array}$ & $\begin{array}{c}0.0182559 \\
(0.1815)\end{array}$ & $\begin{array}{c}0.2951179 \\
(0.4104)\end{array}$ & $\begin{array}{c}0.1471359 \\
(0.1913)\end{array}$ \\
\hline PATRIF2 & $\begin{array}{l}0.44854 \\
(0.2572)\end{array}$ & $\begin{array}{c}0.2593049 \\
(0.1151)\end{array}$ & $\begin{array}{l}0.2043597 \\
(0.0 .2128)\end{array}$ & $\begin{array}{c}0.158603 \\
(0.1763)\end{array}$ & $\begin{array}{c}0.1743042 \\
(0.3464)\end{array}$ & $\begin{array}{c}0.0894829 \\
(0.1942)\end{array}$ \\
\hline PATRIF3 & $\begin{array}{c}0.5325111 \\
(0.3007)\end{array}$ & $\begin{array}{c}0.2883847 \\
(0.1075)\end{array}$ & $\begin{array}{c}0.2401059 \\
(0.2387)\end{array}$ & $\begin{array}{c}0.184333 \\
(0.2020)\end{array}$ & $\begin{array}{c}0.0023776 \\
(0.4022)\end{array}$ & $\begin{array}{c}-0.0011671 \\
(0.1988)\end{array}$ \\
\hline PATRIF4 & $\begin{array}{c}0.7689561 \\
(0.2943)\end{array}$ & $\begin{array}{c}0.3540585 \\
(0.0746)\end{array}$ & $\begin{array}{c}-0.0160559 \\
(0.2425)\end{array}$ & $\begin{array}{c}-0.0108825 \\
(0.1710)\end{array}$ & $\begin{array}{c}0.8148993 \\
(0.3509)\end{array}$ & $\begin{array}{c}0.479606 \\
(0.1955)\end{array}$ \\
\hline PATRIF5 & $\begin{array}{c}0.6093385 \\
(0.2391)\end{array}$ & $\begin{array}{c}0.4274587 \\
(0.1575)\end{array}$ & $\begin{array}{c}0.1306233 \\
(0.1913)\end{array}$ & $\begin{array}{c}0.0896456 \\
(0.1331)\end{array}$ & $\begin{array}{c}0.3531139 \\
(0.3139)\end{array}$ & $\begin{array}{c}0.1664442 \\
(0.1425)\end{array}$ \\
\hline DEPRE & $\begin{array}{c}-0.111287 \\
(0.1361)\end{array}$ & $\begin{array}{c}-0.0972687 \\
(0.0960)\end{array}$ & $\begin{array}{c}0.1247337 \\
(0.1153)\end{array}$ & $\begin{array}{c}0.0908917 \\
(0.0806)\end{array}$ & $\begin{array}{c}-0.0027985 \\
(0.1621)\end{array}$ & $\begin{array}{c}0.0120748 \\
(0.0805)\end{array}$ \\
\hline RELIG & $\begin{array}{c}0.3764411 \\
(0.1502)\end{array}$ & $\begin{array}{c}0.2504094 \\
(0.0881)\end{array}$ & $\begin{array}{c}0.1674103 \\
(0.1155)\end{array}$ & $\begin{array}{c}0.1216362 \\
(0.0883)\end{array}$ & $\begin{array}{c}-0.3576658 \\
(0.1765)\end{array}$ & $\begin{array}{c}-0.1626091 \\
(0.0715)\end{array}$ \\
\hline POUP1A5 & $\begin{array}{c}0.4521587 \\
(0.2219)\end{array}$ & $\begin{array}{c}0.2775097 \\
(0.1113)\end{array}$ & $\begin{array}{c}0.1850573 \\
(0.1923)\end{array}$ & $\begin{array}{c}0.1344222 \\
(0.1535)\end{array}$ & $\begin{array}{c}0.4853595 \\
(0.3155)\end{array}$ & $\begin{array}{c}0.2723738 \\
(0.1866)\end{array}$ \\
\hline POUP5A10 & $\begin{array}{c}0.6249194 \\
(0.2158)\end{array}$ & $\begin{array}{c}0.3710015 \\
(0.1012)\end{array}$ & $\begin{array}{c}0.2000125 \\
(0.1868)\end{array}$ & $\begin{array}{c}0.145947 \\
(0.1469)\end{array}$ & $\begin{array}{c}0.5250213 \\
(0.2907)\end{array}$ & $\begin{array}{c}0.2897584 \\
(0.1662)\end{array}$ \\
\hline POUPAC10 & $\begin{array}{c}0.8646487 \\
(0.2161)\end{array}$ & $\begin{array}{c}0.5228863 \\
(0.1026)\end{array}$ & $\begin{array}{c}0.2007598 \\
(0.1806)\end{array}$ & $\begin{array}{c}0.1375806 \\
(0.1348)\end{array}$ & $\begin{array}{c}0.5726769 \\
(0.2837)\end{array}$ & $\begin{array}{c}0.2958132 \\
(0.1479)\end{array}$ \\
\hline
\end{tabular}




\subsection{Caderneta de poupança}

Ainda conforme os resultados da Tabela 2, a propensão ao risco (RISCO) é um fator estatisticamente significante quando a alternativa de investimento é a caderneta de poupança. A probabilidade de uma mulher com propensão ao risco investir em caderneta de poupança é diminuída em $29,35 \%$. Os efeitos marginais calculados para as mulheres que possuem investimentos em caderneta de poupança são encontrados na Tabela 3.

Na nossa base de dados, a IDADE não é estatisticamente significante para quem investe em caderneta de poupança.

Dentre os coeficientes de ECIVIL só o que representa o grupo de casadas (CAS) é estatisticamente significante. O sinal é negativo. A probabilidade de uma mulher casada investir em caderneta de poupança é diminuída em $24,33 \%$.

No estudo para a variável dependente caderneta de poupança, o patrimônio financeiro (PATRIF) não estatisticamente significante. O fator educação (EDUC) também não é estatisticamente significante.

Os demais fatores relevantes nesse estudo não são estatisticamente significantes com essa base de dados.

\subsection{Outros investimentos}

Conforme os resultados da Tabela 2, assim como no caso de Ações a variável RELIG é estatisticamente significante, porém com sinal negativo. Mulheres que interagem num grupo formado pela mesma religião apresentam $35,77 \%$ menos probabilidade de investir em outros investimentos. Já como era de esperar pessoas que poupam mais têm mais chances de investir em outros investimentos, por exemplo pessoas que poupam mais de $10 \%$ de sua renda tem $57,27 \%$ mais probabilidade de investir em outros investimentos.

Os demais fatores não são estatisticamente significantes em nenhuma combinação de variáveis. Pouco podemos inferir sobre esses dados.

Nesse caso seria melhor se pudéssemos abrir o leque de investimentos. Mas como o foco do estudo é identificar o perfil e características da investidora em ações, deixaremos esse tópico para outro estudo.

Tabela 4: Correlação entres as variáveis relevantes do estudo

\begin{tabular}{lcccccc}
\hline & VIZIN & RELIG & GRUPO & CLUBE & RISCO & DEPRE \\
\hline VIZIN & 1.000000 & & & & & \\
RELIG & -0.152896 & 1.000000 & & & & \\
GRUPO & 0.137437 & -0.113479 & 1.000000 & & & \\
CLUBE & -0.092616 & -0.085621 & 0.028113 & 1.000000 & & \\
RISCO & 0.114922 & 0.062671 & 0.091343 & 0.118521 & 1.000000 & \\
DEPRE & 0.067526 & -0.016446 & -0.008163 & -0.035941 & 0.132594 & 1.000000 \\
\hline
\end{tabular}

\subsection{Interação social}

Os indicadores de interação social não são estatisticamente significantes em nossa análise com exceção de RELIG. Encontrou-se GRUPO estatisticamente significante e com sinal positivo em uma das combinações de regressões tratando-se de outros investimentos, o que pode sugerir que a interação social influencia de alguma forma os investimentos mas, na nossa base de dados, não influencia a participação no mercado de ações, conforme Tabela 5. 
Tabela 5: Indicadores de interação social

\begin{tabular}{lcccc}
\hline & RELIG & CLUBE & GRUPO & VIZIN \\
\hline ACOES & $0.174021^{* *}$ & 0.102377 & 0.083210 & -0.051015 \\
& $(0.076924)$ & $(0.085568)$ & $(0.078732)$ & $(0.078451)$ \\
CPOUP & $0.119479^{*}$ & 0.074618 & $-0.107318^{*}$ & -0.032589 \\
& $(0.068905)$ & $(0.070626)$ & $(0.062912)$ & $(0.066914)$ \\
OUT & $-0.149764^{* *}$ & 0.044307 & $0.206934^{* * *}$ & -0.029267 \\
& $(0.072380)$ & $(0.086521)$ & $(0.073333)$ & $(0.079377)$ \\
\hline \multirow{2}{*}{ Coeficientes das regressões onde a variável dependente é um indicador da alternativa de investimento, } \\
que está na coluna à esquerda. As variáveis independentes são as variáveis de interação social. Os erros \\
\multicolumn{4}{l}{ padrões robustos estão entre parênteses. São 154 observações. Números com * são significantes ao nivel } \\
\multicolumn{7}{l}{ de 10\%, números com ** são significantes ao nível de 5\% e números com *** são significantes ao nível de 1\%. }
\end{tabular}

Hong et alii (2004) citam a importância dos efeitos de peer-group numa variedade de contextos podendo ser em família, vizinhança ou círculos de amizades. Ainda concluíram que os "sociais" são mais propensos a investir no mercado de ações, controlando para fatores como riqueza, raça e educação. Hong et alii (2004) encontraram que os efeitos de sociabilidade são tanto estatisticamente significantes, quanto economicamente importantes. Para os autores a participação no mercado de ações é influenciada pela interação social, isto é, os agentes mais sociais, no senso de interagir mais como os próximos de grupos como igrejas, são mais prováveis de investir no mercado de ações.

\section{CONCLUSÕES}

Os resultados deste estudo evidenciam que além do patrimônio financeiro, o nível de escolaridade, o estado civil, sintomas de depressão e alguns indicadores de interação social são significantes estatisticamente, como sugerido e testado empiricamente por Hong et alii (2004). Também, como proposto por vários outros estudos, a propensão ao risco é determinante na escolha dos investimentos.

O presente estudo sofre um forte viés devido ao tamanho e perfil da amostra. Conclusões muito mais fortes poderão ser obtidas aumentando a amostra. Devido ao alto custo de implementar a base de dados isto ainda não foi feito, esperamos poder fazer isto em pesquisa futura.

\section{BIBLIOGRAFIA}

Bajtelsmit, V. L. \& VanDerhei, J. A. (1996). Risk aversion and retirement income adequacy. In Mitchell, O. S., editor, Positioning Pensions for the Twenty Fisrt Century. University of Pennsylvania Press, Philadelphia.

Banerjee, A. (1992). A simple model of herd behavior. Quarterly Journal of Economics, 107:797-817.

Barber, B. \& Odean, T. (2001). Boys will be boys: Gender, overconfidence and common stock investment. Quarterly Journal of Economics, 116:261-92.

Barberis, N. \& Thaler, R. (2003). Survey of behavioral finance. In Constantinides, G. M., Harris, M., \& Stulz, R., editors, Handbook of the Economics of Finance. Elsevier Science. 
Barsky, R. B., Kimball, M. S., Juster, F. T., \& Shapiro, M. D. (1995). Preference parameters and behavioral heterogeneity: An experimental approach in the health and retirement survey. Technical Report W5213, NBER.

Becker, G. S. (1991). A note on restaurant pricing and other examples of social influences on price. Journal of Political Economy, 99:1109-1116.

Bertrand, M., Luttmer, E., \& Mullainathan, S. (2000). Network effects and welfare cultures. Quarterly Journal of Economics, 115:1019-1055.

Bikhchandani, S., Hirshleifer, D., \& Welch, I. (1992). A theory of fads, fashion, custom and cultural change as informational cascades. Journal of Political Economy, 100:992-1026.

Chiteji, N. S. \& Stafford, F. P. (1999). Portfolio choices of parents and their children as young adults: Asset accumulation by African-American families. The American Economic Review, 89:377-380. Papers and Proceedings of the One Hundred Eleventh Annual Meeting of The American Economic Association.

Choi, J., Laibson, D., \& Metrick, A. (2000). Does the internet increase trading? Evidence from investor behavior in 401(k) plans. Technical Report W7878, NBER.

Croson, R. \& Gneezy, U. (2004). Gender differences in preferences. Journal of Economic Literature. Sep 16.

Durlauf, S. N. (2001). A framework for the study of individual behavior and social interactions. Sociological Methodology, 31:47-87.

Ellison, G. \& Fudenberg, D. (1995). Word of mouth communication and social learning. Quarterly Journal of Economics, 110:93-125.

Granovetter, M. (1983). The strength of weak ties: A network theory revisited. Sociological Theory, 1:201-233.

Hinz, R. P., McCarthy, D. D., \& Turner, J. A. (1996). Are women conservative investors? Gender differences in participant-direct pension investments. In Mitchell, O. S., editor, Positioning Pensions for the Twenty First Century. University of Pennsylvania Press, Philadelphia.

Hong, H., Kubik, J., \& Stein, J. C. (2004). Social interactions and stock market participation. Journal of Finance, 59:137-63.

Jianakoplos, N. \& Bernasek, A. (1998). Are women more risk averse? Economic Inquiry, 36:620-630.

Levy, M. (2003). Are rich people smarter? Journal of Economic Theory, 110:42-64.

Mandrian, B. C. \& Shea, D. D. (2001). The power of suggestion: Inertia in 401(k). Participations and savings behavior. Quarterly Journal of Economics, CXVI:1149-87.

Scheinkman, J. A. (2005). Social Interactions. Princeton University.

Shiller, R. J. (1995). Conversation, information and herd behavior. The American Economic Review, 85:181185.

Spencer, S. J., Steele, C. M., \& Quinn, D. M. (1999). Stereotype threat and women's math performance. Journal of Experimental Social Psychology, 35:4-28.

Vissing-Jorgensen, A. (2000). Towards an explanation of household portfolio choice heterogeneity: Nonfinancial income and participation cost structures. Technical report, University of Chicago.

Welch, I. (1992). Sequential sales, learning and cascades. The Journal of Finance, 47:695-732. 


\section{A. APÊNDICE}

\section{Dados Pessoais}

1. Idade?

2. Estado Civil? ( )solteira ( )casada ( )separada/divorciada ( )viúva

3. Filhos? () não tem () tem filhos

4. Educação? ( ) $1^{\circ}$ grau completo () $2^{\circ}$ grau completo () $3^{\circ}$ grau completo () pós graduação

5. Ocupação? () Não trabalha ( )Trabalha Profissão? _ tabela de profissões

6. Sua infância foi: () com dificuldades financeiras ( ) sem problemas de dinheiro

7. Estado? _ Tabela de estados _ Cidade?

8. Cor? () Branca ( ) Mulata ( ) Negra () Amarela

\section{Patrimônio}

9. Faixa de renda? () até 400 ()de 400 a 2.000 () 2.000 a 4.000 ( ) 4.000 a 6.000 ()acima de 6.000

10. Quanto guarda mensalmente? () Não guardo () entre 1 e $5 \%$ () entre $5 \%$ e $10 \%$ ( )mais da $10 \%$

11. Como você aplica seu dinheiro: ( )caderneta de poupança () CDB ( )fundo de renda fixa ( ) fundos de ações ( ) ações ( ) Outros? Quais? _ tabela de outras opções

12. Patrimônio financeiro? () até 5.000 ()de 5.000 a 25.000 () 25.000 a 50.000 ( ()50.000 a 75.000 ()acima de 75.000

13. Imóvel que mora hoje? ( )alugado ( ) próprio. Valor aproximado

14. Possui automóvel: ( ) não ( ) sim

\section{Interação Social}

15. Visita parentes ou amigos? () sim ( )não

16. Visita seus vizinhos? () sim ()não

17. Frequenta algum serviço religioso? () sim ( )não

18. Pratica algum esporte? () sim ( )não

19. Frequenta uma academia ou Clube? () sim ( )não

20. Participa de algum grupo ou associação? () sim ( )não

\section{Características}

21. Se você receber a oferta de um novo trabalho tão bom quanto o que você tem atualmente, mas com $50 \%$ de chance de dobrar sua renda e $50 \%$ de reduzi-la em $1 / 3$, você aceitaria a proposta? ( ) sim ( ) não 22. Na semana passada sentiu-se deprimida? ( ) sim ( ) não

\section{Tecnologia}

23. Possui e-mail? ( ) sim ( ) não

24. Possui computador? ( ) sim ( ) não

Questionário formulado com base no artigo "Social Interaction and Stock-Market Participation" do The Journal of Finance - Vol. LIX, No. 1 - February 2004 e no questionário da Health and Retirement Study (HRS) administrado pelo Institute for Social Research da University of Michigan. Os questionários estão disponíveis no site www. umich. edu $/ \sim \mathrm{hrswww} /$. 
Tabela A-1: Resumo das estatísticas - Total da amostra

\begin{tabular}{|c|c|c|c|c|}
\hline & Média & Desvio-padrão & Mín. & Máx \\
\hline IDADE & 42,1 & $(10,3000)$ & 23 & 41 \\
\hline IAB25 & 0,0129 & $(0,1135)$ & 0 & 1 \\
\hline I25A35 & 0,2532 & $(0,4362)$ & 0 & 1 \\
\hline I35A45 & 0,3051 & $(0,4619)$ & 0 & 1 \\
\hline I45A55 & 0,2987 & $(0,4591)$ & 0 & 1 \\
\hline IAC55 & 0,0974 & $(0,2974)$ & 0 & 1 \\
\hline \multicolumn{5}{|l|}{ ECIVIL } \\
\hline SOL & 0,2792 & $(0,4500)$ & 0 & 1 \\
\hline CAS & 0,4740 & $(0,5009)$ & 0 & 1 \\
\hline SEP & 0,2077 & $(0,4070)$ & 0 & 1 \\
\hline VIU & 0,0389 & $(0,1941)$ & 0 & 1 \\
\hline FILHO & 0,5584 & $(0,2481)$ & 0 & 1 \\
\hline \multicolumn{5}{|l|}{ EDUC } \\
\hline EDUC2 & 0,0519 & $(0,2226)$ & 0 & 1 \\
\hline EDUC3 & 0,3766 & $(0,4861)$ & 0 & 1 \\
\hline EDUC4 & 0,5649 & $(0,4973)$ & 0 & 1 \\
\hline TRAB & 0,9480 & $(0,2226)$ & 0 & 1 \\
\hline PROFIS & 0,3246 & $(0,4697)$ & 0 & 1 \\
\hline PROBFIN & 0,3896 & $(0,4982)$ & 0 & 1 \\
\hline EST & 0,8311 & $(0,3758)$ & 0 & 1 \\
\hline COR & 0,9415 & $(0,2353)$ & 0 & 1 \\
\hline \multicolumn{5}{|l|}{ RENDA } \\
\hline R0 & 0,0000 & $(0,0000)$ & 0 & 0 \\
\hline R0A2 & 0,0779 & $(0,2689)$ & 0 & 1 \\
\hline R2A4 & 0,1818 & $(0,3869)$ & 0 & 1 \\
\hline R4A6 & 0,2337 & $(0,4246)$ & 0 & 1 \\
\hline RAC6 & 0,4870 & $(0,5014)$ & 0 & 1 \\
\hline POUPO & 0,8441 & $(0,3638)$ & 0 & 1 \\
\hline \multicolumn{5}{|l|}{ INVEST } \\
\hline CPOUP & 0,2993 & $(0,4595)$ & 0 & 1 \\
\hline $\mathrm{CDB}$ & 0,1753 & $(0,3814)$ & 0 & 1 \\
\hline FRF & 0,5194 & $(0,5012)$ & 0 & 1 \\
\hline ACOES & 0,6780 & $(0,4688)$ & 0 & 1 \\
\hline OUT & 0,2727 & $(0,4468)$ & 0 & 1 \\
\hline \multicolumn{5}{|l|}{ PATRIF } \\
\hline PATRIF1 & 0,0454 & $(0,2089)$ & 0 & 1 \\
\hline PATRIF2 & 0,1168 & $(0,3223)$ & 0 & 1 \\
\hline PATRIF3 & 0,0844 & $(0,2789)$ & 0 & 1 \\
\hline PATRIF4 & 0,0909 & $(0,2884)$ & 0 & 1 \\
\hline PATRIF5 & 0,5779 & $(0,4955)$ & 0 & 1 \\
\hline IMOV & 0,8116 & $(0,3922)$ & 0 & 1 \\
\hline AUTO & 0,8571 & $(0,3510)$ & 0 & 1 \\
\hline SOCIAL & 1,0000 & $(0,0000)$ & 0 & 1 \\
\hline VIZIN & 0,4675 & $(0,5005)$ & 0 & 1 \\
\hline RELIG & 0,6883 & $(0,4646)$ & 0 & 1 \\
\hline ESP & 0,5986 & $(0,4917)$ & 0 & 1 \\
\hline CLUBE & 0,6283 & $(0,4848)$ & 0 & 1 \\
\hline GRUPO & 0,6442 & $(0,4803)$ & 0 & 1 \\
\hline RISCO & 0,3591 & $(0,4814)$ & 0 & 1 \\
\hline DEPR & 0,5519 & $(0,4989)$ & 0 & 1 \\
\hline EMAIL & 0,9934 & $(0,0808)$ & 0 & 1 \\
\hline COMP & 0,9933 & $(0,0813)$ & 0 & 1 \\
\hline \multicolumn{2}{|c|}{ Tamanho da amostra } & & 154 & \\
\hline
\end{tabular}

